\title{
Topiktests und Topikpositionen im Ungarischen: Ergebnisse und theoretische Bewertung einer Korpusanalyse
}

\begin{abstract}
Der vorliegende Beitrag hat zum Ziel, bestimmte Eigenschaften von Ausdrücken im ungarischen Topikfeld mit Hilfe von Korpusuntersuchungen aus einer neuen Perspektive zu beleuchten. Die bekanntesten Topikdefinitionen und die für die Bestimmung von Topiks vorgeschlagenen Tests der Fachliteratur werden zusammengefasst und die wichtigsten Vorschläge zur Behandlung der Topikposition des ungarischen Satzes werden besprochen. Ich präsentiere aus den Tests hervorgegangene Beobachtungen, die es erzwingen, gewisse bisherige Vorstellungen über die syntaktische Struktur des ungarischen Satzes neu zu überdenken. Eine davon betrifft die Verfeinerung der Zusammenhänge zwischen den Positionen von Nominalphrasen im ungarischen Topikfeld und den TopikTests. Außerdem diskutieren wir Gegenbeispiele zu informationsstatusbasierten Abfolgebeschränkungen und Zugänglichkeitsprinzipien der Topikfeldbesetzung bei Zustandsänderungsverben.
\end{abstract}

Abstract (ung.): A tanulmány célja az, hogy a magyar mondat topik mezőjében elhelyezkedő elemek bizonyos tulajdonságait korpuszvizsgálatokra alapozva új perspektívából mutassa be. Áttekintjük a legfontosabb topikdefiníciókat és a topikok azonosítására javasolt teszteket, valamint a magyar mondat topik pozíciójával kapcsolatos legfontosabb eddigi megállapításokat. Bemutatjuk korpuszvizsgálataink során tett azon megállapításainkat, amelyek a magyar mondat szintaktikai szerkezetével kapcsolatos bizonyos eddigi elképzelések újragondolását teszik szükségessé. Ezek egyrészt új összefüggésekre mutatnak rá a topik mezőben elhelyezkedő főnévi kifejezések pozíciójával és a topik tesztek teljesítésével kapcsolatban. Másrészt, ellenpéldákat mutatunk be az információs státusszal összefüggő javasolt sorrendi megszorításokra, valamint az állapotváltozást leíró igéket tartalmazó mondatok topik pozícióinak kitöltésére vonatkozóan. 


\section{Einführung ${ }^{1}$}

Im generativen Modell der Syntax des ungarischen Satzes von Katalin É. Kiss (vgl. É. Kiss 1987, 1994, 2002, 2010), das als der am weitesten anerkannte Ansatz gelten kann, tragen bestimmte präverbale Positionen die Namen informationsstruktureller Kategorien wie Topik oder Fokus. Bisher sind aber die Details der Korrespondenz zwischen diesen Positionen und den informationsstrukturellen Eigenschaften nicht ausreichend untersucht. Während dem Zusammenhang zwischen unmittelbar präverbaler Fokusposition und Fokussierung große Aufmerksamkeit geschenkt wurde, ist der zwischen Topikposition und Topikfunktion wesentlich weniger intensiv studiert worden.

Der vorliegende Beitrag setzt sich zum Ziel, die Eigenschaften der Elemente in der ungarischen Topikposition mit Hilfe von Untersuchungen, die an dem im Rahmen des EuroGr@mm-Projektes erstellten Wikipedia-Korpus vorgenommen wurden, eingehender $\mathrm{zu}$ analysieren. Diesem Ziel entsprechend werde ich in Abschnitt 2 einen Überblick über die bekanntesten Topikdefinitionen und die für die Bestimmung der Topiks vorgeschlagenen Tests der Fachliteratur geben. In Abschnitt 3 fasse ich gewisse Feststellungen der Fachliteratur, die sich mit der Topikposition des ungarischen Satzes und den Elementen in dieser Position befassen, zusammen und werte sie aus. In Abschnitt 4 erläutere ich die Zielsetzungen der Korpusanalysen bezüglich des Ungarischen und die wichtigsten Ergebnisse. Das Ziel dieser Korpusuntersuchungen war, gewisse Behauptungen der Fachliteratur zu testen. Während des Testens wurden mehrere Beobachtungen gemacht, die es erzwingen, gewisse bisherige Vorstellungen über die syntaktische Struktur des ungarischen Satzes neu zu überdenken. Eine davon betrifft systematische Asymmetrien zwischen Nominalphrasen im sogenannten Topikfeld des ungarischen Satzes bzgl. positionsabhängiger Erfüllung einschlägiger Topiktests. Außerdem konnten Gegenbeispiele zu informationsstatusbasierten Abfolgebeschränkungen (Lipták 2011) und Zugänglichkeitsprinzipien der Topikfeldbesetzung bei Zustandsänderungsverben (É. Kiss 2005) gefunden werden. Abschnitt 5 enthält einige Schlussfolgerungen.

1 Ich bedanke mich bei Péter Bassola für die Übersetzung und Hans-Martin Gärtner für die Überarbeitung der Übersetzung sowie bei den Herausgebern dieses Bandes für hilfreiche Anmerkungen. 


\section{Topikdefinitionen in der Fachliteratur und ihre Anwendbarkeit}

\subsection{A(boutness)-Topik}

Es gibt zahlreiche Arbeiten, die sich empirisch und konzeptuell mit der Informationsstruktur auseinandersetzen. Im Weiteren wird kurz erläutert, wie die zuverlässigsten Tests zur Bestimmung der Topikausdrücke in den unterschiedlichen Sprachen aussehen. Im vorliegenden Beitrag werde ich mich in erster Linie auf die theoretische Herangehensweise der Studien von Krifka (2007) und Krifka/ Musan (2012), sowie auf Beiträge des Bandes Krifka/Musan (Hgg.) (2012) stützen, ferner auf die Hinweise, die Dipper/Götze/Skopeteas (2007) zur Analyse bestimmter Texte von bestimmten Sprachen geben.

Die in der Fachliteratur am meisten anerkannte Topikdefinition stammt von Reinhart (1981), gestützt auf Vorschläge von Strawson (1964). Laut dieser Definition ist das Topik im Grunde die Entität, über die der Satz informiert. Die eher technische Formulierung der obigen Intuition stützt sich auf den Begriff der von Stalnaker (1978) eingeführten Kontextmenge ('context set'), welcher (vereinfacht) die Menge der von den Teilnehmern des Gesprächs als wahr angenommenen Behauptungen bezeichnet, wobei jede Folgeäußerung diese Menge anreichert. Der Annahme folgend, dass die von den Äußerungen ausgedrückte Information (falls möglich) in der Kontextmenge so repräsentiert ist wie in einem Karteikartensystem, betrachtet Reinhart (1981) das Topik als die Entität (oder die Menge der Entitäten), auf deren Karteikarte die von den weiteren Teilen des Satzes getragene Information (der Kommentar) in der Kontextmenge erfasst wird. Die der obigen Definition entsprechenden Entitäten werden in der Fachliteratur auch als Aboutness-Topiks (im Folgenden „A-Topiks“) bezeichnet, um den Unterschied zu Vorstellungen, welche die Topiks vor allem als 'gegeben'/'alt' betrachten, zu betonen. (Zum Vergleich der beiden Topikauffassungen s. Frey 2004 und die dort zitierte Literatur.) Die Bestimmung der Topikausdrücke in einem Satz wirkt sich auf eine Reihe weiterer Domänen aus.

Einerseits sichert, wie Roberts (2011) es ausführlich darstellt, die systematische Topikwahl die Kohärenz des Textes und somit zugleich die erfolgreichere Verarbeitung der Information (s. z.B. Krifka/Musan 2012 zum Begriff 'common ground management'). Außerdem beeinflusst die Topikwahl die Präsuppositionen des Satzes und liefert Defaultreferenten für die Pronomina (Reinhart 1981), womit sie zur Bestimmung der Wahrheitsbedingungen des Satzes beiträgt. Die obigen Mechanismen können aber formal nur beschrieben werden, wenn im Falle einzelner Sätze eindeutig festgestellt werden kann, was ihr Topik ist. 
Reinhart (1981) schränkt die potenziellen Topiks auf die Entitäten ein, auf die sich eine der Konstituenten des Satzes bezieht. ${ }^{2}$ Sätze, die keine (potenziell elidierten) Topikkonstituenten enthalten, werden in der einschlägigen Literatur als thetisch, d.h. Sätze mit informationsstrukturell ungeteilten Behauptungen bezeichnet. Mit solchen Sätzen werden nicht von einem Individuum, sondern von der Situation selbst Aussagen gemacht. Demgegenüber heißen Aussagen in Sätzen mit Topik kategorisch, d.h. in zwei Teile - in einen, ein Individuum einführenden und einen darüber etwas aussagenden Teil - geteilte Aussagen. (Zur Unterscheidung thetisch-kategorisch und deren grammatischer Auswirkung s. u.a. Drubig 1992; Jäger 2001; Kuroda 1972; Ladusaw 1994; Marty 1884; Reinhart 1981; Sasse 1987.)

Die Fachliteratur nimmt bezüglich zweier, miteinander zusammenhängender Fragen unterschiedliche Standpunkte ein. Einerseits geht es darum, ob das Topik innerhalb eines isolierten Satzes (zusammen mit der Intonation des Satzes) bestimmt werden kann, oder ob die Bestimmung des Topiks auch vom Kontext abhängt. Andererseits stellt sich die Frage, ob ein Satz mehrere Topiks haben kann.

Im Zusammenhang mit der ersten Frage meint Reinhart (1981: 53) (auf Grundlage des Englischen), dass im Normalfall ein in unterschiedlichen Kontexten und potenziell mit unterschiedlichen Intonationen geäußerter Satz unterschiedliche Topiks haben kann. Ausgenommen ist aber der Fall, dass er grammatische Mittel enthält, die speziell zur Auszeichnung des Topiks dienen (wie z.B. die Linksversetzung), da in diesem Fall das Topik des Satzes konstant ist. Sasse (1987) zeigt mit der Gegenüberstellung der folgenden Beispiele (1a-b), dass im Deutschen Akzente auf Subjekt und Verb eine eindeutig kategorische Aussage festlegen, während bei alleinigem Akzent auf dem Subjekt eine thetische Aussage vorliegt. (Zu englischsprachigen Beispielen s. Chafe 1974.)

a. Das BRAThendl ist angebrannt.

b. Das BRAThendl ist ANgebrannt.

(Sasse 1987: 529)

Nach Reinhart (1981: 56) kann ein Satz höchstens ein (eventuell koordiniertes) Topik haben. ${ }^{3}$ Diese Auffassung kann aber bei Sprachen zu Widersprüchen führen, in denen gleichzeitig mehrere Konstituenten mit grammatischen Merkmalen

2 Diese Anforderung muss in dem Ungarischen ähnlichen Pro-drop-Sprachen natürlich gelockert werden, damit auch die von den elidierten Konstituenten bzw. gewissen gebundenen Morphemen auf indirekte Weise eingeführten Referenten als Topiks betrachtet werden können.

3 Dieser Meinung sind außer ihr noch Molnár (1991, 1998), Vallduví (1990) und Jacobs (2001) bzgl. des Deutschen. Entgegengesetzter Meinung sind É. Kiss (2002), Lasnik/Saito (1992), Kornfilt 
versehen werden können, die für Topiks charakteristisch sind, wie beispielsweise die Versetzung in präverbale Position im Ungarischen (ausführlicher s. unten in Abschnitt 3) oder die Versetzung in die Topikposition im Mittelfeld im Deutschen (Frey 2004). U.a. auch deshalb nehmen Frey (2004) und Endriss (2009) den Standpunkt ein, dass das Topik eines Satzes unabhängig vom Text immer eindeutig festgestellt werden kann, und ein Satz auch mehrere Topiks haben kann.

Einen Zwischenstandpunkt vertreten Krifka (2007) sowie Krifka/Musan (2012), nach denen ein Satz zwar mehrere Topiks haben kann, in diesem Fall aber ihr Beitrag zur Kontextmenge so modelliert wird, dass die Informationen für beide Referenten gemeinsam auf einer Karteikarte erfasst werden.

Unter den Autoren, die mit der obigen allgemeinen Definition des A-Topiks einverstanden sind, gibt es auch solche, die es für eine notwendige Bedingung des Topiks (Gundel 1985; Vallduví 1990) oder für eine prototypische Begleiterscheinung (Chafe 1976; Reinhart 1981) halten, dass der Referent des Topikausdrucks aus dem vorangehenden Diskurs als bekannt oder unabhängig vom Diskurs für den Hörer identifizierbar sein sollte. Krifka/Musan (2012: 28) betonen dahingegen, dass auch eine den obigen Bedingungen nicht entsprechende (m.a.W. eine neu eingeführte) Entität Topik des Satzes sein kann. Ähnlicher Meinung sind auch Endriss (2009), Frey (2004) und Sasse (1987).

\subsection{Tests zur Bestimmung von Topiks bzw. Topikkonstituenten}

Reinhart (1981: 64) stellt zwei Tests zur Ermittlung von Topikkonstituenten vor. Der eine ist die Ersetzbarkeit durch einen Satz, der zusätzlich eine bestimmte linksversetzte Konstituente, nämlich As for X 'Was X betrifft', enthält.

Der andere ist die Einbettungsmöglichkeit in einen Matrixsatz wie He said about $X$ that ... 'Er sagte von X, dass ...'. Reinhart gibt selbst zu, dass der erste Test deshalb, weil Linksversetzung eher Mittel eines Topikwechsels ist als Mittel der Einführung eines neuen Topiks, nicht allgemein anwendbar ist, beispielsweise nicht für spezifische oder generische Indefinita. ${ }^{4}$

(1997) und Rizzi (1997), die es für möglich halten, dass in englischen, ungarischen, türkischen und italienischen Sätzen eine unbegrenzte Anzahl von Topiks vorkommen.

4 Ward (1985: 23) plädiert dafür, dass auch der zweite Test nicht für die gesamte Gruppe der potenziellen Topikausdrücke angewendet werden kann; die Belege unter (i) und (ii) können nicht durch die unter (i') und (ii') ersetzt werden.

(i) Some computation he may not be able to carry out in his head.

(i') \# He says about some computation that he may not be able to carry it out in his head. 
Gundel (1985) schlägt zwei andere Tests vor. Nach dem ersten muss ein Satz mit einem Topikausdruck $X$ eine korrekte Antwort auf die Frage What about X? 'Was ist mit $X$ ?' sein können. Nach dem zweiten Test muss der Satz $S$ mit dem Topikausdruck $X$ durch einen Satz ersetzt werden können, den wir dadurch bekommen, dass $S$ durch Speaking of $X$ 'Was $X$ anbetrifft' eingeleitet wird. ${ }^{5}$

Roberts (2011) wendet die obigen vier Tests auf Sätze mitten in absatzlangen Texten an. Der Testtext von Roberts, dessen ursprüngliche Quelle Ward (1985: 73) ist, steht unter (2). Unter (3) wird veranschaulicht, wie die Transformationen in den obigen Tests nach Roberts auf das Akkusativobjekt (Coppola) des zweiten Satzes angewendet werden können. (\# markiert Sätze, deren Einsetzung in den Originaltext zu pragmatischer Unangemessenheit führen würde.)

(2) Then Tom Cruise went to work for Francis Ford Coppola, on this spring's semisuccessful film version of "The Outsiders". Coppola he found to be "just like one of the guys. And he totally trusted me. He let me go anywhere I wanted to go with the character...” (Philadelphia Inquirer: 8-C, 9/1/83, „His star is rising, but his feet remain firmly on the ground.“, zitiert nach Ward 1985: 73)

(3) (i) About Coppola, he said that he found him to be...

(ii) \# What about Coppola? He found him to be...

(iii) \# As for Coppola, he found him to be...

(iv) \# Speaking of Coppola, he found him to be... (Roberts 2011: 1914f.)

Laut Roberts treten die folgenden Probleme auf. Der What about- und der As forTest implizieren einen Kontrast zwischen Coppola und einer anderen salienten Diskursentität, was aber der Text in (2) nicht hergibt. Die Anwendung der Konstruktion Speaking of $X$ weist nicht auf einen solchen Kontrast hin, aber sie präsupponiert, dass Coppola, obwohl er nicht Gegenstand des Gespräches ist, vorerwähnt wurde, und signalisiert gleichzeitig den Wechsel zum Reden über ihn. Roberts ist der Meinung, der Grund für den degradierten Status von (3iv) läge darin, dass dieser Wechsel schon im Vorgängersatz angezeigt wurde.

Die Konstruktion About $X$ führt dieselbe Präsupposition ein, was in diesem Fall nicht zu Widersprüchen führt, wodurch (3i) aus pragmatischer Sicht angemessen ist. Für ersterwähnte Topiks sind aber die zwei zuletzt genannten Tests unangemessen.

(ii) Just the slightest pressure he found difficult to handle.

(ii') \# He said about just the slightest pressure that he found it difficult to handle.

5 Ward (1985: 23) hält den ersten von Gundels Tests ebenfalls für problematisch. 
Im Gegensatz zu Reinhart, Gundel, Ward und Roberts nimmt Frey (2004) an, dass Topiks in einem Satz unabhängig vom Kontext bestimmbar sind. Nach seinem Vorschlag muss man $X$ dann als Topikausdruck des Satzes $S$ betrachten, wenn wir - sobald Ich erzähle dir etwas von $X$ vor S gestellt wird - einen kohärenten Text bekommen. Der Autor plädiert dafür, dass sich über der Basisposition der Satzadverbialia im Mittelfeld des deutschen Satzes eine Topikposition befindet, wo alle Mittelfeldtopiks, und nur diese, platziert sind. Das folgende Beispiel zeigt, dass der Satz (4a), wo Maria sich in dieser höheren Position befindet, dem obigen Kriterium genüge tut. Bei (4b) ist dies nicht der Fall:

(4) Ich erzähle dir etwas über Maria.

a. Nächstes Jahr wird Maria wahrscheinlich nach London gehen.

b. \# Nächstes Jahr wird wahrscheinlich Maria nach London gehen.

(Frey 2004: 158)

Frey ist der Meinung, dass, weil mehr als eine Konstituente in der Topikposition im Mittelfeld vorkommen kann, in einem deutschen Satz mehr als ein Topik möglich ist. Derselben Meinung ist auch Endriss (2009), die - wie Frey - Topiks isolierter Sätze untersucht.

Ziel der Arbeit von Götze et al. (2007) ist es, zur Analyse der Informationsstruktur von Texten unterschiedlicher Sprachen methodische Hilfestellung zu leisten. Für die Identifikation von Topiks schlagen sie die Tests unter (5) und (6) vor, von denen der erste unter (5) mit dem von Frey verwendeten Test identisch ist, während die anderen beiden eine Variante der von Reinhart und Gundel stammenden und oben bereits beschriebenen Tests zu sein scheinen.

(5) Eine NP $X$ ist das A-Topik des $X$ enthaltenden Satzes $S$, wenn

- $S$ eine natürliche Fortsetzung des folgenden Satzes ist:

Let me tell you something about $X$. 'Lass mich etwas von $X$ sagen.'

- $S$ eine passende Antwort auf die folgende Frage ist:

What about $X$ ? 'Was ist mit $X$ ?'

- $S$ auf natürliche Weise in den folgenden Satz transformiert werden kann:

Concerning $X, S^{\prime}$, 'Was $X$ betrifft, $S$ '

oder den folgenden:

Concerning $X, S^{\prime}$, 'Was $X$ betrifft, $S$ "

wo $S^{\prime}$ sich von $S$ nur insofern unterscheidet, dass darin $X$ von einem entsprechenden Pronomen vertreten wird. ${ }^{6}$ (Götze et al. 2007: 165)

6 Bei Sätzen, die generische indefinite Ausdrücke im Singular oder Adverbialquantoren enthalten, muss das erste Vorkommen von $X$ in den obigen Tests durch den entsprechenden 
(6) Ein spezifisches Indefinitum $X$ ist das A-Topik eines $X$ enthaltenden Satzes, wenn $S$ nach der folgenden Transformation natürlich klingt:

- Ersetze in $S$ den indefiniten Artikel von $X$ durch dies oder jenes

- Stelle vor den entstandenen $S^{\prime}$ die Formel: Concerning $X, S^{\prime}$, 'Was $X$ betrifft, $S^{\prime \prime}$

(Götze et al. 2007: 165)

Cook/Bildhauer (2013) überprüfen die Anwendbarkeit der von Götze et al. (2007) vorgeschlagenen Tests anhand der Analyse deutschsprachiger Texte. In ihrer Studie, in der zwei Annotierer anhand der obigen Tests die Beispiele aus einem Korpus analysieren sollten, haben sie im Hinblick auf die Sätze - die vier bestimmte Verben mit unterschiedlichen Argumentstrukturen enthielten - recht unterschiedliche Ergebnisse erhalten. Diskrepanzen ergaben sich sowohl bezüglich der Frage, ob die untersuchten Sätze überhaupt ein Topik haben, als auch bezüglich der Frage, welche Ausdrücke - Subjekt-NP, Akkusativ-NP, gewisse deiktische adverbiale Ausdrücke wie hier oder dann - als topikal betrachtet werden müssen.

Laut der Hypothese von Cook/Bildhauer (2013) kann jeder Satz höchstens ein Topik haben (wie schon von Reinhart 1981 vorgeschlagen). Bei dessen Auswahl spielt eine Rolle, wie der Informationsstatus des Topikreferenten ist (gegeben, zugänglich, neu, s. Götze et al. 2007 und Abschnitt 4.2). Somit kann sich das Topik eines Satzes je nach Kontext ändern. ${ }^{7}$

Darüber hinaus fanden Cook/Bildhauer (2013) den ersten Test unter (5) schwer anwendbar, da die Eingliederung des Satzes Lass mich dir etwas von $X$ sagen! den Informationsstatus des von $X$ denotierten Referenten verändert, was ihrer Meinung nach für die Bestimmung des Satztopiks von Belang ist. Insgesamt halten die Autoren die von Götze et al. (2007) vorgeschlagenen Tests für nicht ausreichend operationalisierbar. Zudem äußern sie sich skeptisch bzgl. der Frage, ob die Instruktionen für die Bestimmung der Elemente der Informationsstruktur unabhängig von der untersuchten Sprache formuliert werden können.

Auf Grund des obigen Überblicks zeichnen sich folgende Resultate ab. In Anbetracht der Arbeiten von Ward (1985) und Roberts (2011) lässt sich annehmen, dass die Anwendbarkeit der Tests zur Ermittlung von A-Topiks bei Sätzen innerhalb eines größeren Diskurses von verschiedenen Textfaktoren abhängt. Daher bedeutet die Tatsache, dass eine bestimmte Konstituente einen bestimmten Topiktest nicht erfüllt, nicht notwendigerweise, dass sie nicht topikal ist. Die Literatur liefert keinen Test, der aus einer Menge möglicher A-Topiks innerhalb

Ausdruck im Plural und ohne Artikel ersetzt werden.

7 Unter den von den Autoren als Illustration präsentierten Sätzen gab es keine, in denen irgendeine potenzielle Topikkonstituente in der Mittelfeldtopikposition von Frey (2004) vorkam. 
beliebiger Satz-Kontext-Paare das Reinhart'sche A-Topik identifiziert. Für eine operationalisierbare Prozedur zur satzinternen Identifikation von A-Topiks müssen wir wohl auf die Definition von Frey (2004) zurückgreifen, die kontextunabhängig funktioniert, und das gleichzeitige Auftreten mehrerer A-Topiks zulässt. In Anbetracht dessen, dass vorliegender Beitrag in ungarischen Sätzen nur die Topikeigenschaften von Konstituenten untersucht, die in einer 'Topikposition' platziert sind, scheint die von Frey (2004) vorgeschlagene Strategie besonders gut geeignet zu sein.

Leider finden sich in der einschlägigen Literatur keine zuverlässigen Tests zur Auffindung thetischer Sätze. ${ }^{8}$ Deshalb scheint die einzig mögliche Strategie zu sein, Sätze dann als thetisch anzusehen, wenn sie keine Konstituenten enthalten, die Freys Topiktest erfüllen.

\subsection{Kontrastive und rahmensetzende Topiks}

Krifka (2007) sowie Krifka/Musan (2012) verstehen unter kontrastiven Topiks (im Folgenden „K-Topiks“) solche A-Topiks, die bestimmte Eigenschaften von A-Topiks und Fokus kombinieren: Sie führen A-Topiks mit Alternativenbezug (topikinternem Fokus) ein, wie beispielsweise die Subjekt-NPn im Antwortteil des folgenden Dialogs: ${ }^{9}$

$$
\begin{aligned}
& \text { A: What do your siblings do? } \\
& \text { B: [My } \left.[\text { SIster }]_{\text {Focus }}\right]_{\text {Topic }}[\text { studies MEDicine }]_{\text {Focus }} \text {, } \\
& \text { and [my [BROther } \left.]_{\text {Focus }}\right]_{\text {Topic }}[\text { is working on a FREIGHT ship }]_{\text {Focus }} \text {. }
\end{aligned}
$$

(Krifka 2007: 32, (44))

Laut diesen Autoren zeigt ein K-Topik an, dass die vom (lokalen) Satz formulierte Behauptung weniger Information enthält, als in der konkreten Situation, Beantwortung einer Kontextfrage wie (7A), zu erwarten wäre.

8 Gécseg/Kiefer (2009) betrachten (in Anlehnung an Puskás 2000: 58) diejenigen Sätze als Ausdrucksformen von thetischen Behauptungen, die auf die Frage Was ist passiert? antworten. Ich werde diesen Test jedoch nicht anwenden, weil er in Wirklichkeit nur prüft, ob wir es mit Satzfokus zu tun haben (Krifka/Musan 2012). Dieses Problem wurde schon von Molnár (2011) angemerkt.

9 Zur ausführlicheren Analyse der semantisch-pragmatischen Eigenschaften von K-Topiks s. noch u.a. Büring (1997, 2003), van Hoof (2003), Kadmon (2001) und Wagner (2008), zu den Eigenschaften des ungarischen K-Topiks u.a. Gyuris (2009) und Molnár (1998). 
Wegen dieser semantisch-pragmatischen Eigenschaften zählen Krifka (2007) sowie Krifka/Musan (2012) die K-Topiks - zusammen mit den rahmensetzenden Topiks - im Folgenden „R-Topiks“ - zu den sog. Delimitierern. Laut Götze et al. (2007: 167f.) bestimmen R-Topiks den Rahmen, bezüglich dessen die Hauptprädikationen des gegebenen Satzes gedeutet werden sollen. Häufig wird durch sie die Zeit oder der Ort des vom weiteren Teil des Satzes beschriebenen Ereignisses oder Zustands spezifiziert, weshalb sie typischerweise durch Temporal- oder Lokaladverbialia ausgedrückt werden. Solche Adverbialia gelten aber nach den genannten Autoren nicht automatisch als R-Topiks, sondern nur dann, wenn sie sich in gewissen syntaktischen Positionen - typischerweise in der linken Peripherie befinden. Die von ihnen bestimmten Orte bzw. Zeitpunkte/Zeitintervalle sind bereits häufig Teile des gemeinsamen Redehintergrunds oder sie können daraus leicht erschlossen werden. Einen weiteren Typ von R-Topiks stellen sogenannte 'Domänenadverbiale' dar, wie körperlich in (8).

Körperlich geht es Peter gut.

(Jacobs 2001: 655)

Götze et al. (2007) machen zugleich darauf aufmerksam, dass es im Falle der R-Topiks keine direkte Prädikationsrelation zwischen dem Topik und dem Denotat des Restteils des Satzes gibt. Daraus folgt, dass nach ihrer Auffassung ein Ausdruck nicht gleichzeitig ein A-Topik und ein R-Topik sein kann. Allerdings bieten sie keine Tests zur Auswahl der R-Topiks, die den Tests in (5) und (6) ähnlich wären.

Jacobs (2001: 656) zeigt an bestimmten Beispielen, dass die Auswahl von R-Topiks die Wahrheitsbedingungen des Satzes beeinflussen kann, die von A-Topiks nicht. Wenn dies als allgemeine Eigenschaft von R-Topiks betrachtet werden kann, dann liegt folgender Test zu deren Identifizierung nahe: Ein Ausdruck $X$ - der die Eigenschaften von R-Topiks in jeder anderen Hinsicht erfüllen kann - kann als R-Topik des Satzes $S$ dienen, wenn aus der Wahrheit von $S$ nicht automatisch die Wahrheit der Variante von $S$ ohne $X$ folgt. (Aus In Ungarn sind alle Politiker korrupt folgt nicht Alle Politiker sind korrupt.) Diese Methode hat allerdings das Problem, dass sie voraussetzt, dass die Menge der in Frage kommenden R-Topiks mit irgendeiner anderen (z.B. syntaktischen) Methode bestimmbar sein muss. Ein weiteres Problem ist, dass sie bestimmte von anderen Autoren eindeutig als R-Topik betrachteten Konstituenten, wie z.B. das Adverb gestern in (9) (s. Götze et al. 2007), ausschließen würde. Es ist leicht einzusehen, dass aus der Wahrheit von (9B) eindeutig folgt, dass Hans geheiratet hat (vgl. Davidson 1967).

(9) A: Ist gestern etwas Interessantes passiert?

B: Gestern hat Hans geheiratet. 
Maienborn (2001) unterscheidet unter den lokalen Modifikatoren die sog. rahmensetzenden Modifikatoren. Sie nimmt an, dass diese „lokationale Beschränkungen“ (wörtl. „locative constraints“) für unterpezifizierte Referenten ausdrücken. Ein Beispiel findet sich in (10), wo der Modifikator die Quantifikationsdomäne von die meisten Schwäne einschränkt. Ein anderer Fall liegt in (11) vor, wo es keinen sprachlich ausgedrückten Anker für diese Funktion des Lokativs gibt. In einem Kontext, wo es um Maßnahmen bezüglich öffentlicher Räume geht, kann man annehmen, dass der Modifikator die Menge dieser Räume einschränkt.

(10) a. In Australien sind die meisten Schwäne schwarz.

b. In Frankfurt ist die Einwohnerzahl gestiegen.

(Maienborn 2001: 232, (91))

(11) In London war Trafalgar Square abgesperrt.

(Maienborn 2001: 233, (92))

Obwohl die Theorie von Maienborn (2001) eine den Intuitionen gut entsprechende Interpretation der lokalen R-Topiks zulässt, ist mir nicht klar, wie sie zur generellen Ermittlung von R-Topiks bestimmter Sätze ohne Kontext nutzbar gemacht werden kann.

\section{Die ungarische Topikposition und ihre informationsstrukturellen Charakteristika: ein Blick in die Fachliteratur}

\subsection{Topikposition und Topikfunktion in den Arbeiten von Katalin É. Kiss}

In theoretischen Arbeiten zum Ungarischen verwendet man den Ausdruck Topik in Anlehnung an die Arbeiten von Katalin É. Kiss (z.B. É. Kiss 1987, 1994, 2002, 2010) meistens zur Bezeichnung der in einer gewissen syntaktischen Position der Satzstruktur erscheinenden Konstituenten. Die Topikposition, die in der obigen Theorie den Spezifikator der Projektion TopP in der linken Peripherie der Struktur des Hauptsatzes bezeichnet, trägt deshalb den Namen dieses informationsstrukturellen Begriffs, weil die Ausdrücke an dieser Stelle laut der Autorin die 
Entität benennen, von der der Satz handelt. ${ }^{10}$ Diese Argumentation wird von der Tatsache unterstützt, dass in Topikpositionen nur sog. referierende Ausdrücke vorkommen können. In Anlehnung an die Beobachtungen von Reinhart (1981: 66), dass Indefinita nur in ihrer generischen oder spezifischen Lesart Topiks bezeichnen können, behauptet É. Kiss, dass die Indefinita in der Topikposition nur eine generische oder spezifische Lesart haben können. Dabei versteht sie unter spezifischer Lesart (in Anlehnung an Enç 1991), dass das Indefinitum ein Individuum bezeichnet, welches Element einer bereits früher eingeführten Menge von Entitäten ist. ${ }^{11}$
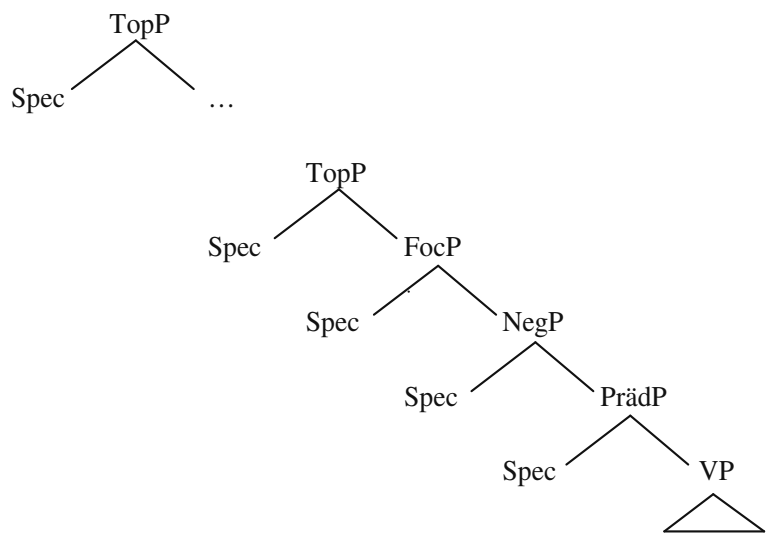

In der Satzstruktur des Ungarischen folgt der Reihe der Konstituenten in Topikpositionen, die ich im Weiteren auch als Topikbereich oder Topikfeld bezeichne, der Rest des Satzes, den É. Kiss als Prädikat bezeichnet. É. Kiss modelliert dies so, dass in der hierarchischen Struktur die iterierbare TopP die PrädP dominiert, welche als erweiterte Projektion des Verbausdrucks (VP) zu betrachten ist. Bei nicht-neutralen Sätzen erscheinen über der PrädP weitere Projektionen, z.B. NegP (mit der Negationspartikel in ihrem Spezifikator), oder FocP (mit dem präverbalen Identifikationsfokus in ihrem Spezifikator), die aber noch zum Prädikatsteil des Satzes gehören. Die obengenannten Projektionen sind in (12) (in Anlehnung an É. Kiss 2010²) illustriert.

10 Diese Beobachtung stammt ursprünglich von Brassai (1860, 1963-65).

11 Es ist anzumerken, dass zusätzlich zu dieser Auffassung von Spezifizität, von Farkas (1994) als „partitive Spezifizität“ bezeichnet, noch mindestens zwei weitere Ansichten in der Literatur gängig sind. „Epistemische Spezifizität“ (vgl. Fodor/Sag 1982) betrifft die Frage, ob ein Sprecher einen bestimmten Referenten intendiert, und „skopale Spezifizität“ hängt davon ab, ob das Indefinitum im Skopus eines Quantors, der Negation oder eines intensionalen Prädikats interpretiert wird.

12 Die Distributivquantoren, denen in früheren Theorien gesonderte präverbale Projektionen zugewiesen wurden (s. z.B. É. Kiss 2002 oder Szabolcsi 1997), werden laut É. Kiss (2010) an die Projektionen PrädP, NegP oder FocP adjungiert. 
Zur Bestimmung der Grenze zwischen Topikfeld und Prädikat schlägt É. Kiss zwei Tests vor. Der eine ist prosodischer Art: Die erste Hauptkonstituente des Prädikats ist diejenige Konstituente, auf die der erste obligatorische Akzent des ungarischen Satzes fällt. Nach É. Kiss ist dies gleichzeitig der stärkste grammatisch determinierte Akzent.

Der zweite Test hängt mit der Position der Satzadverbialia zusammen: Da diese, wie in (13) gezeigt, vor, zwischen oder unmittelbar nach den Konstituenten in Topikpositionen erscheinen können, muss jede Konstituente, die einem Satzadverbial vorangeht, ein Topik sein.

(13) a. Sajnos az új autónak Péter [PrädP megnyomta az elejét.] eider ART neu Auto.DAT P. eindrückte ART Vorderseite.AKK 'Leider hat Peter das neue Auto an der Vorderseite verbeult.'

(É. Kiss 1998b: 27)

b. Az új autónak állítólag Péter [PrädP megnyomta az elejét.] ART neu Auto.DAT angeblich P. eindrückte ART Vorderseite.AKK 'Das neue Auto hat Peter an der Vorderseite angeblich verbeult.'

(É. Kiss 1998b: 27)

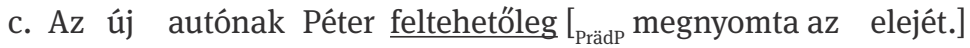
ART neu Auto.DAT P. vielleicht eindrückte ART Vorderseite.AKK 'Das neue Auto hat Peter vielleicht an der Vorderseite verbeult.'

(É. Kiss 1998b: 27)

In Anbetracht dessen, dass die etablierte phonologische Beschreibung des ungarischen Satzes (s. u.a. Kálmán/Nádasdy 2004; Varga 2002) dagegen spricht, dass er nur einen Hauptakzent haben kann, werde ich mich im Weiteren bei der Bestimmung der Topik-Prädikat-Grenze eher auf den zweiten Test stützen.

É. Kiss (2002: 13) nimmt an, dass der Satz, wenn mehr als eine Topikkonstituente im Topikfeld auftritt, eine Prädikation über ein Paar (n-Tupel) von Individuen ausdrückt. ${ }^{13}$

13 Keiner der bisher besprochenen Topiktests ist allerdings in der Lage, abzuleiten, dass das Topik von i) aus dem Paar az új autónak und Péter besteht. ii) illustriert die Anwendung des What about $X$ ?-Tests und iii) die von Frey (2004) vorgeschlagene Methode:

i) Az új autónak Péter megnyomta az elejét. ART neu Auto.DAT P. eindrückte ART Vorderseite.AKK 'Peter hat das neue Auto an der Vorderseite verbeult.'

ii) A: Mi történt Péterrel és az új autóval? was passierte Peter.mit und ART neu Auto.mit 'Was ist mit Peter und dem neuen Auto passiert?'

B: \# Az új autónak Péter megnyomta az elejét. 
Im Zusammenhang mit der Feststellung, dass Indefinita nur in spezifischer oder generischer Lesart in der Topikposition vorkommen können, meint É. Kiss (2002), dass im folgenden Satz der satzinitiale nominale Ausdruck nur dann in Topikposition stehen kann, wenn er ein Element einer Menge von Autos denotiert, die bereits früher eingeführt wurde. ${ }^{14}$

$\begin{array}{lllll}\text { Egy } & \text { autó megállt } & \text { a } & \text { házunk } & \text { előtt. } \\ \text { ein } & \text { Auto anhielt } & \text { ART } & \text { Haus.unser } & \text { vor }\end{array}$

'Ein Auto hat vor unserem Haus angehalten.'

(É. Kiss 2002: 10)

Für den Vorschlag zur Besetzung und Interpretation der Topikposition könnte der folgende Satz problematisch sein, da hier scheinbar ein nicht-spezifischer indefiniter Ausdruck ${ }^{15}$ die Topikposition besetzt: ${ }^{16}$

Valami leesett a tetőről.
etwas runterfiel ART Dach.von
'Etwas ist vom Dach runtergefallen.'

(É. Kiss 2002: 11)

Laut É. Kiss (2002) erfüllt jedoch die satzinitiale Konstituente in (15) das Spezifizitätskriterium, da hier eine Eigenschaft von einem Individuum prädiziert wird, das den Diskursteilnehmern aus dem nichtverbalen Kontext bekannt ist. Ich glaube allerdings nicht, dass die Autorin in dieser Sache richtig liegt, da (15) nicht in einer Situation geäußert wird, in der der Sprecher von einer bestimmten, ihm mental präsenten, Entität aussagen möchte, dass sie vom Dach gefallen sei, sondern z.B. in einer Situation, in der die Gesprächsteilnehmer die Quelle eines bestimmten Geräuschs eruieren. Der Satzadverbial-Test zeigt auch ein negatives Ergebnis: valószínüleg 'wahrscheinlich' kann dem satzinitialen Indefinitum nicht nachfolgen, vgl. den Kontrast zwischen (16a) und (16b).

\footnotetext{
iii) Mondok valamit az új autóról és Péterről. sage etwas.AKK ART neu Auto.über und Peter.über 'Ich sage dir etwas über das neue Auto und Peter.'

\# Az új autónak Péter megnyomta az elejét.
}

14 Sonst würde es sich wie ein Quantor am Anfang des Prädikats an die PrädP adjungiert befinden. 15 Nicht-Spezifizität gilt hier sowohl im Sinne von partitiver als auch im Sinne von epistemischer Spezifizität.

16 Valami 'etwas' kann hier nicht die Fokusposition des Satzes ([Spec, FocP]) besetzen, da eine Füllung dieser Position die Inversion von Präfix und Verb verlangen würde. 
(16)
a. *Valami valószínüleg leesett a tetőtől. etwas wahrscheinlich runterfiel ART Dach.von
'Etwas ist wahrscheinlich vom Dach runtergefallen.'
b. Valószínüleg valami leesett a tetőtől.
'Wahrscheinlich ist etwas vom Dach runtergefallen.'

Diese Tatsache könnte bedeuten, dass sich valami nicht in der Topikposition des Satzes, sondern - an die PrädP adjungiert, ähnlich wie die anderen Distributivquantoren - vor dem Prädikat befindet. Für distributive Quantoren gilt allerdings, dass auf sie nur andere distributive Quantoren, ein präverbaler Fokus oder das Verb folgen können. (16a) wird aber akzeptabel, wenn wir das Satzadverbial valószínüleg gegen das Temporaladverb tegnap austauschen, wie in (17). ${ }^{17}$
Valami tegnap leesett a tetőtől. etwas gestern runterfiel ART Dach.von
'Etwas ist gestern vom Dach runtergefallen.'

Der Unterschied zwischen (16a) und (17) kann am einfachsten erklärt werden, wenn wir annehmen, dass das bisher als homogen betrachtete Topikfeld in zwei Teile geteilt wird. Dies ist in (18) zu sehen: Hier dominieren die iterierbaren Top1PPositionen die wiederum iterierbaren Top2P-Projektionen.
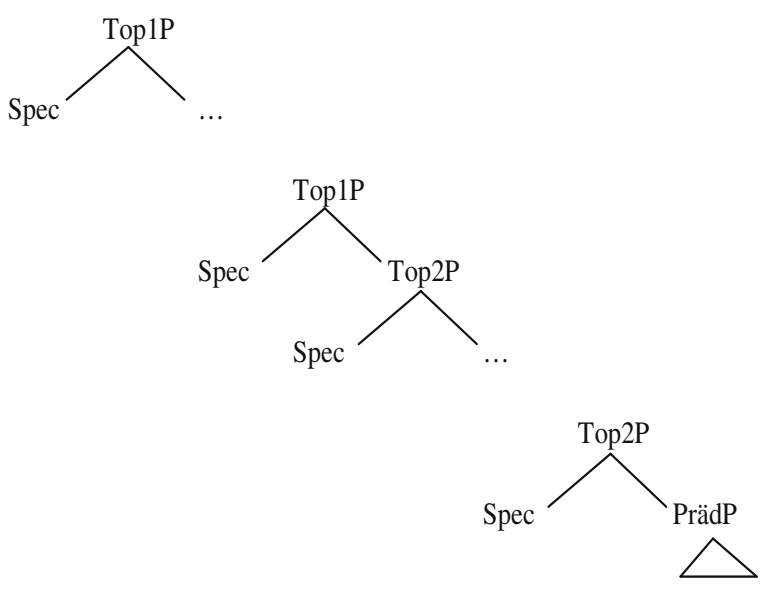

17 In (17) kann tegnap nicht in der präverbalen Fokusposition sein, da es sonst unmittelbar dem Verb, das mit dem Verbpräfix le invertiert ist, vorangehen müsste. 
Auf Grundlage dieser Struktur können wir annehmen, dass spezifische Indefinita sowohl in Spec,Top1P als auch in Spec,Top2P auftauchen können, nicht-spezifische dahingegen nur in Spec,Top2P. Der Wortstellungskontrast zwischen Temporal- und Lokaladverbialen auf der einen und Satzadverbialen allgemein auf der anderen Seite, lässt sich durch die Annahme erklären, dass erstere frei an Top1Pn und Top2Pn adjungieren können, während alle anderen Satzadverbialia nur an Top1P und die höchste der Top2Pn adjungiert sein dürfen. In Abschnitt 4 werde ich auf weitere Asymmetrien bezüglich der syntaktisch-semantischen Eigenschaften der Konstituenten innerhalb des Topikfeldes hinweisen. ${ }^{18}$

Sprechen wir kurz über die Syntax der potenziellen R-Topiks im Ungarischen. Die temporalen und lokalen Ausdrücke, die an der linken Peripherie des Satzes stehen, wie beispielsweise die ersten beiden Konstituenten des Satzes (19), befinden sich nach É. Kiss in Topikpositionen und erfüllen dabei auch die semantischen Eigenschaften der 'logischen Subjekte' (d.h. der weitere Teil des Satzes handelt von ihnen; wie oben ausgeführt, spreche ich hier von A-Topiks). ${ }^{19}$

Magyarországon az idén kétszer is megáradtak a folyók.
Ungarn.auf ART dieses.Jahr zweimal auch fluteten ART Flüsse
In Ungarn traten die Flüsse in diesem Jahr auch schon zweimal über die Ufer.

É. Kiss (2002: 18, (20))

Da wir, wenn der Satz (20) vor den obigen Satz gestellt wird, einen kohärenten Diskurs erhalten, kann gesagt werden, dass die im Lokaladverbial enthaltene NP Ungarn das Frey'sche (2004) Kriterium für A-Topiks erfüllt. ${ }^{20}$

$\begin{array}{lll}\text { Mondok } & \text { valamit } & \text { Magyarországról. } \\ \text { sage } & \text { etwas.AkK } & \text { Ungarn.über } \\ \text { 'Ich erzähle etwas über Ungarn.' }\end{array}$

18 Gyuris/Mády (2014) plädieren auf Grund der topologischen Eigenschaften der A-Topiks und K-Topiks für die Unterscheidung weiterer Teilbereiche innerhalb des Topikbereiches.

19 Von weiteren Ausdrücken, deren Entsprechungen die Fachliteratur zu den R-Topiks zählt (z.B. physisch, finanziell u.a.), spricht É. Kiss nicht, aber es ist anzunehmen, dass sie diese zu den Satzadverbialia zählen und somit im (bei ihr undifferenzierten) Topikfeld platzieren würde.

20 Obige Klassifizierung verursacht in der Theorie von Jacobs keine Probleme. Er plädiert dafür, dass mehrere R-Topiks - und dabei insbesondere linksversetzte Konstituenten im Deutschen eine Adresse ,in the speaker-hearer knowledge where the information carried by [the sentence] has to be stored at the moment of the utterance“ (Jacobs 2001: 650) spezifizieren. Wie dem aufmerksamen Leser nicht entgangen sein dürfte, handelt es sich hier auch um die Definitionseigenschaft von A-Topiks. Es ist zugleich nicht klar, welcher Status in der Theorie von Götze et al. (2007), die es ausschließen, dass eine Konstituente gleichzeitig ein A-Topik und ein R-Topik bezeichnet, dem satzeinleitenden Lokaladverbial von (19) zugeordnet werden soll. 
Das folgende Beispiel zeigt, dass die Entscheidung, ob ein einen Ort oder Zeitpunkt/Zeitintervall bezeichnender Ausdruck den obigen A-Topiktest erfüllt, nicht nur von der Bedeutung des Ausdrucks oder der syntaktischen Struktur des Satzes abhängt, sondern auch von gewissen semantisch-pragmatischen Eigenschaften bzw. von Weltwissen (also davon, inwieweit die von dem Prädikatsteil des Satzes ausgedrückte Eigenschaft fähig ist, in einem bestimmten Kontext zur allgemeinen Charakterisierung des potenziellen Topiks zu dienen):
a. Mondok
valamit
Budapestről.
erzähle
etwas.AKK
Budapest.über
'Ich erzähle etwas über Budapest.'
b. Budapesten jól érezte magát II. Erzsébet.
Budapest.IN wohl fühlte sich.AKK Elizabeth II
'In Budapest fühlte sich Königin Elizabeth II. wohl.'
c. \# Budapesten jól érezte magát János.
\# 'In Budapest fühlte sich János wohl.'

Es ist nach É. Kiss (2005) keinesfalls beliebig, welche Konstituenten (Argumente) im ungarischen Satz Topikpositionen einnehmen und welche im Prädikatsbereich des Satzes bleiben. Die Verallgemeinerungen formuliert sie für vier Verbklassen: die Verbklassen der Zustandsänderung, der Ortsänderung, des Aufenthaltes in einem Ort sowie des mentalen oder physischen Zustandes. Von diesen finden wir im Wikipedia-Korpus in genügender Menge Sätze nur mit Verben der Zustandsänderung (z.B. megír 'schreiben', lenyír ‘abschneiden' u.a.). Deshalb konzentrieren wir uns auf die Verallgemeinerungen, die diese betreffen. Nach É. Kiss, die sich in ihrer Analyse nur mit isolierten Sätzen befasst, kann das Argument mit der thematischen Rolle Thema in den obigen Sätzen von dem Prädikatsbereich in den Topikbereich des Satzes kommen, wenn ein anderes, einen neuen Referenten einführendes Argument in der VP bleibt, ein Argument einen Kontrast ausdrückt - indem es in die Fokusposition gelangt - oder das Verb emphatisch betont bzw. negiert wird. In Abschnitt 4 werde ich noch auf die Validität dieser Voraussagen eingehen.

\subsection{Gécseg/Kiefer (2009)}

Auch Gécseg/Kiefer (2009) machen darauf aufmerksam, dass temporale Adverbialia bestimmten nonspezifischen Indefinita nachfolgen können, bemerken aber den Unterschied zwischen temporalen und Satzadverbialia in (16)-(17) nicht. Sie 
merken an, dass zu den Konstituenten, die sich im Ansatz von É. Kiss in [Spec, TopP] befinden, nicht nur topikalische Ausdrücke sondern auch nichtspezifische Indefinita gehören müssen. Gécseg/Kiefer (2009) nehmen an, dass die Konstituenten in [Spec, TopP] ein gemeinsames semantisches Merkmal haben, nämlich Referenz auf Dinge, über die der Satz unabhängig vom Kontext etwas aussagt. Solche Konstituenten nennen die Autoren „logische Subjekte“ des Satzes. ${ }^{21}$ Dementsprechend schlagen sie vor, die TopP-Projektionen in LsubjP-Projektionen umzubenennen. Was das Verhältnis dieser Positionen zu den A-Topik-Konstituenten anbelangt, können letztere sowohl in [Spec, LsubjP] als auch in postverbalen Positionen vorkommen, beispielsweise in Sätzen mit Individuenebenen-Prädikaten, die keine thetische Aussage machen können (in Anlehnung an Ladusaw 1994)..$^{22,23}$

Die obige Auffassung führt zu gewissen Widersprüchen. Während die Autoren an einer Stelle (Gécseg/Kiefer 2009: 587) die von Brentano (1874) und Marty (1918) gegebene Definition der thetischen Aussagen übernehmen, nach der diese als ungegliederte Aussagen betrachtet werden können, sagen sie an anderer Stelle, dass Sätze mit ausgefüllten logischen Subjektpositionen ebenfalls thetische Aussagen formulieren können. Die Autoren gehen nicht darauf ein, ob im Falle von mehreren logischen Subjekten die das logische Subjekt definierenden Eigenschaften zutreffen, und wenn ja, inwiefern. ${ }^{24}$

21 „The logical subject of the sentence is an element referring to an individual or set of individuals of which something is predicated.“ (Gécseg/Kiefer 2009: 607).

22 Nach Gécseg/Kiefer (2009: 610) ist das in postverbaler Position stehende Subjekt des folgenden Satzes mit einem Individuenebenen-Prädikat ein Topik:

(i) Nagyon szeretik a gyerekeim a palacsintát.

sehr mögen ART Kinder.meine ART Palatschinken.AKK

'Meine Kinder mögen Palatschinken sehr.'

23 Der folgende neutrale Satz mit flacher Prosodie (Kálmán 1985) und ein NP-Argument in TopP/ LsubjP-Position, der nach allgemeiner Beurteilung einen Satzfokus hat, drückt den Autoren zufolge ebenfalls eine thetische Aussage aus:

(i) A 'kutyátok 'megharapta a 'lányomat.
ART Hund.euer gebeisste ART Tochter.meine.AKK
'Euer Hund hat meine Tochter gebissen.'

Diese Auffassung steht in deutlichem Widerspruch zur Meinung von Kálmán, der annimmt, dass neutrale Sätze Topikkonstituenten haben können.

24 Während unserer Korpusuntersuchung konnten wir die Behauptung leider nicht überprüfen, ob nonspezifische Indefinita sich vor dem Prädikatsbereich des Satzes befinden können, da nonspezifische Indefinita weder vor Satzadverbialia, noch vor solchen definiten Nominalausdrücken vorkamen, von denen wir mit Sicherheit wissen, dass sie in Topikposition platziert sind. Im ungarischen Nationalkorpus (www.corpus.nytud.hu/mnsz) konnten im Topikbereich keine 


\subsection{Lipták (2011)}

Laut Lipták erfüllen die Elemente, die nach É. Kiss in bestimmten Topikpositionen stehen können, eindeutig die Funktion des A-Topiks in Reinhart'schem Sinne. Nach der Autorin können gleichzeitig auch mehrere A-Topiks in einem Satz stehen, ihr Informationsstatus kann sowohl gegeben als auch neu sein, obwohl die Ausdrücke mit dem Informationsstatus neu immer nach denen mit Status gegeben vorkommen. Ihrer Meinung nach wird dies vom zweiten Konjunkt des folgenden Beispiels illustriert. ${ }^{25}$

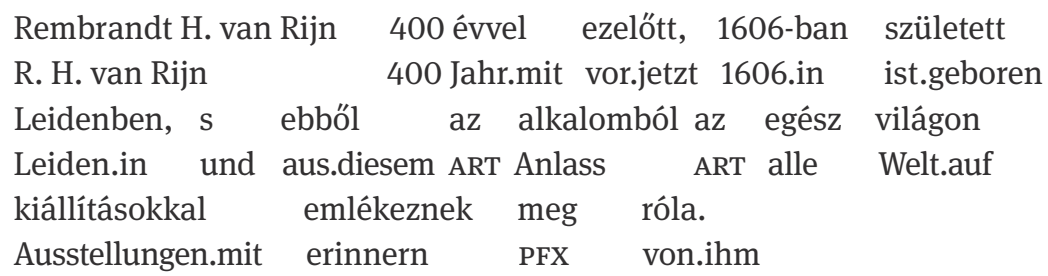

'Rembrandt H. van Rijn ist vor 400 Jahren 1606 in Leiden geboren, und aus diesem Anlass wurden zu seinen Ehren in aller Welt Ausstellungen abgehalten.'

(Lipták, 2011: 6)

Im Zusammenhang mit der Analyse des obigen Satzes ergeben sich zahlreiche Probleme. Zum Einen: Der Ausdruck in aller Welt erfüllt den Frey'schen A-Topik-Test nicht; er kann eher als R-Topik eingestuft werden. Zum Anderen: Der Ausdruck in aller Welt ist kein gutes Beispiel zur Einführung eines neuen Diskursreferenten. Götze et al. (2007) würden ihn bezüglich des Informationsstatus eher als zugänglich betrachten.

Wie É. Kiss betrachtet Lipták die temporalen und lokalen Adverbialia, die nach ihr rahmensetzend (wörtlich 'scene-setting') fungieren, als in jeglicher Reihenfolge zwischen die Konstituenten in Topik-Positionen einfügbar. ${ }^{26}$

Nach diesem Überblick über die Fachliteratur gehe ich im folgenden Abschnitt auf die Ergebnisse der Korpusanalyse ein.

Belege von nicht-spezifischen NPn mit Indefinitartikel, auf die Satzadverben wie sajnos 'leider', szerencsére 'glücklicherweise’, or valószínüleg 'möglicherweise' folgen, gefunden werden.

25 Dies steht der Auffassung von Gécseg/Kiefer (2009) gegenüber, nach der die Reihenfolge der Konstituenten in Topik- bzw. in Positionen des logischen Subjekts unabhängig von ihrem Informationsstatus ist.

26 Lipták macht außerdem in Bezug auf die Reihenfolge der A-Topiks und der K-Topiks interessante Feststellungen, auf die hier aus Platzmangel nicht eingegangen werden kann. 


\section{Korpusanalyse}

\subsection{Ziel der Untersuchungen}

Die Untersuchungen am ungarischen Wikipedia-Korpus ${ }^{27}$ befassten sich mit der Analyse der informationsstrukturellen Eigenschaften von Konstituenten im Topikbereich, die mit Hilfe von (nach É. Kiss festgestellten) syntaktischen Kriterien (Abschnitt 3.1) leicht zu identifizieren sind. Ziel der Untersuchungen war es, die einzelnen, in der syntaktischen Fachliteratur formulierten Behauptungen zu testen. Ganz konkret sollten die folgenden Fragen beantwortet werden:

(23) i) Wenn die Topikfelder mehrere nominale Ausdrücke (potenzielle A-Topiks) enthalten, verfügen sie alle über A-Topik-Eigenschaften? Mit welchen Eigenschaften korreliert ein A-Topik, wenn nicht alle Ausdrücke darüber verfügen? (Syntaktische Rolle, Informationsstatus, topologische Position im Topikbereich u.a.)

ii) Besteht der von Lipták (2011) beschriebene Zusammenhang zwischen der Reihenfolge und dem Informationsstatus der A-Topiks, d.h., gehen die Ausdrücke mit dem Informationsstatus gegeben immer denen mit dem Informationstatus neu voran?

iii) Welche Feststellungen können in Bezug auf die Reihenfolge der A- und der potenziellen R-Topiks (vor allem der temporalen und lokalen Adverbialia) gemacht werden?

iv) Können Sprecherpräferenzen im Zusammenhang damit beobachtet werden, wie viele Konstituenten mit welcher Eigenschaft in diese Position kommen können, mit besonderer Rücksicht auf den Standpunkt von É. Kiss (1987, 1994, 2002 und 2010), nach dem die Besetzung des Topikfeldes optional ist, und auf die Feststellungen von É. Kiss (2005) bzgl. der Frage, welche Argumente im Falle der Verben der Zustandsänderung im Prädikatsbereich des Satzes bleiben müssen? ${ }^{28}$

27 Benutzt wurde das getaggte Korpus Wiki_HU2-öffentlich (10 Millionen Wörter), das im Rahmen des EuroGr@mm-Projekts auf Grundlage ungarischer Wikipedia-Texte zu Forschungszwecken aufgebaut wurde.

28 Unter dem Gesichtspunkt der Struktur des Topikbereichs wäre es noch interessant gewesen, die relative Reihenfolge der A- bzw. der R-Topiks und der Satzadverbialia zu untersuchen. Das zur Verfügung stehende Korpus ermöglichte dies leider nicht, da die Wikipedia-Texte - von ihrer Gattung her - (ihr Ziel ist es, faktische Informationen möglichst bündig zu vermitteln) relativ wenige Satzadverbialia enthalten. 


\subsection{Ablauf der Untersuchungen}

Die obengenannten Ziele sollten durch die Analyse ungarischer Hauptsätze erreicht werden, welche über einen Topikbereich mit zwei Konstituenten verfügen.

Wir wollten die Sätze ausschließen, in denen unter den präverbalen Konstituenten präverbaler (Exhaustiv-/Identifikations-)Fokus oder Verbmodifizierer zu finden sind. Es wurde nach Sätzen gesucht, wo mindestens zwei, höchstens acht Nomina mit Artikeln oder Adverbien vor der Verbpartikel zu finden sind. (Dies schließt aus, dass ein präverbaler Ausdruck als Fokus oder Verbmodifizierer zu betrachten ist.) Von der so erhaltenen Liste wurden diejenigen Sätze entfernt, in deren Topikbereich sich koordinierte NPn befanden, wo die Konstituenten im präverbalen Bereich aus irgendeinem Grunde eine komplexe Konstituente bildeten, wo einer der Ausdrücke eine gelöschte Teilkonstituente enthielt, oder wo deshalb, weil ein Teil der präverbalen Ausdrücke in den Prädikatsbereich des Satzes gelangte (z.B. als Distributivquantor oder als Prädikatsadverbial), nicht mindestens zwei Konstituenten im Topikbereich geblieben sind. Unter den so verbliebenen Sätzen befanden sich 452 mit zwei, sieben mit drei Konstituenten, und ein Satz im Topikbereich mit vier Konstituenten. In Anbetracht der niedrigen Zahl der letzten beiden Kategorien, werde ich mich im Weiteren auf die Sätze konzentrieren, die Topikbereiche mit jeweils zwei Konstituenten hatten.

Ob die Elemente des Topikbereiches eines Satzes $S$ über die Charakteristika der A-Topiks verfügen, wurde auf die von Frey (2004) vorgeschlagene Weise (s. Abschnitt 2.1) getestet. Es wurde untersucht, ob man, wenn der Satz Ich sage etwas über $X$ dem Satz $S$ vorangestellt wird (wo $X$ dem potenziellen Topikausdruck entspricht), einen kohärenten Text erhält. ${ }^{29}$

Der Informationsstatus der Elemente des Topikbereiches wurde in dem von Götze et al. (2007) vorgeschlagenen theoretischen Rahmen, der drei unterschiedliche Kategorien von Referenten unterscheidet, untersucht. Auf die Refenten mit dem Status gegeben wurde im vorangehenden Teil des Textes explizit verwiesen. Auf die Referenten mit dem Status zugänglich wurde zwar nicht explizit verwiesen, aber sie sind aus dem außersprachlichen Kontext zu identifizieren, nämlich durch das Weltwissen des Hörers, oder dadurch, dass der bewusste Referent in irgendeinem Verhältnis zu einem früher eingeführten Referenten steht. Falls der Referent keine der obigen Bedingungen erfüllt, ist sein Status neu.

29 Es muss betont werden, dass der Test nicht unbedingt verlangt, dass der Satz Ich erzähle etwas über $X$ auch in den dem Satz $S$ vorangehenden Text einfügbar sein muss. 


\subsection{Ergebnisse der Untersuchungen}

\subsubsection{A-Topiks im Topikbereich}

Im Zusammenhang mit der Frage (23i), die sich auf die Eigenschaften, welche für die A-Topiks der nominalen Ausdrücke im Topikbereich charakteristisch sind, bezog, konnten die folgenden überraschenden Beobachtungen gemacht werden. Subjekt-NPn, die im Topikbereich an der zweiten oder einer späteren Stelle auftreten, bestehen aus systematischen Gründen den oben beschriebenen Topiktest nicht. Sehen wir uns als Illustration die Texte (24) bis (27) an, deren jeweiliger zweiter Satz ein Hauptsatz aus dem Wikipedia-Korpus ist:
a. Mondok
valamit
a
veséről.
erzähle
etwas.AKK
ART
Niere.über
'Ich erzähle etwas über die Niere.'

b. \# A penicillineket a vese eltávolítja a szervezetből. ART Penicillin.PL.AKK ART Niere entfernt ART Organismus.aus

\# 'Penicillin wird aus dem menschlichen Organismus durch die Niere abgeführt.'

(25)
a. Mondok valamit a zsinatról.
erzähle etwas.AKK ART Synode.über

'Ich erzähle etwas über die Synode.'

b. \# XXIII. Jánost a zsinat felfüggesztette.

XXIII. J.AKK ART Synode suspendierte

\# 'Johannes XXIII. wurde von der Synode suspendiert.'

(26)
a. Mondok
valamit
Bartókról.
erzähle
etwas.AKK
Bartók.über

'Ich erzähle etwas über Bartók.'
b. \# A fölkérést
ART Einladung.AKK
Bartók elfogadta.
B.
annahm
\# 'Die Einladung hat Bartók angenommen.'
a. Mondok
valamit
Krúdy Gyuláról.
erzähle
etwas.AKK
K. Gy.über
'Ich erzähle etwas über Gyula Krúdy.'
b. \# A fogadó hangulatát
Krúdy Gyula
ART Gasthof Stimmung.POSs.AKK
K. Gy.
megörökítette
verewigte 
az Aranykéz utcai szép napok címü müvében.

ART Aranykéz Strasse.von schön Tage betitelt Werk.sein.in

\# 'Die Stimmung des Gasthofs wurde von Gyula Krúdy in seinem Werk

„Die schönen Tage in der Aranykéz-Straße“ verewigt.'

Sehen wir uns nun zum Vergleich einen Satz an, in dem das Subjekt in der ersten Position des Topikbereichs und in der zweiten Position ein anderes Satzglied (das Akkusativobjekt) zu finden ist. Die Tests zeigen, dass in diesen Sätzen beide präverbalen Konstituenten über die Eigenschaft von A-Topiks verfügen:
a. Mondok
valamit
Mátyás
királyról.
erzähle
etwas.AKK
Matthias
König.über

'Ich erzähle etwas über König Matthias.'

b. Mátyás király a lázadást elfojtotta.

Matthias König ART Aufruhr.AKK niederschlug

'König Matthias hat den Aufruhr niedergeschlagen.'
a. Mondok valamit a lázadásról.
erzähle etwas.AKK ART Aufruhr.über
'Ich erzähle etwas über den Aufruhr.'
b. Mátyás király a lázadást elfojtotta.
'König Matthias hat den Aufruhr niedergeschlagen.'

Die obigen Resultate, die die Aufmerksamkeit auf ein in der Fachliteratur bisher noch nicht beobachtetes Phänomen lenken, bedürfen einerseits gezielter, möglichst auf der Basis eines größeren Korpus durchzuführender, weiterer Untersuchungen; sie erzwingen es andererseits, die theoretischen syntaktischen Folgen zu eruieren. Die Daten können auf den ersten Blick unter den zur Verfügung stehenden Theorien am ehesten vielleicht mit den Ansichten von Horváth (1986: 20-22) in Einklang gebracht werden, nach der das Subjekt des ungarischen Satzes in präverbaler Position generiert wird (im Gegensatz zur Behauptung von É. Kiss, nach der dies in postverbaler Position geschieht). Die sonstigen präverbalen Konstituenten kommen durch eine, Topikalisierung genannte, Bewegung zu ihren Oberflächenpositionen, wovon sich die oberste links von der Subjektposition befindet. Diese Transformation ist prinzipiell auch auf präverbale Subjekte anwendbar, die somit links von anderen präverbalen Konstituenten auftauchen können. Wenn die obige Bewegung auf rekursive Weise Projektionen zustande bringt, die das Subjekt dominieren, dann können wir erklären, warum die Subjekt-NPn in der zweiten und dritten Position die Topiktests nicht bestehen. Sie machen nämlich die Topikalisierungstransformation nicht mit, sondern bleiben 
in ihrer Ausgangsposition. (Dass das Subjekt optional auch in postverbaler Stellung vorkommen kann, erklärt Horvath mit Hilfe einer Operation, die das Subjekt nach hinten versetzt.) Ich möchte zugleich betonen, dass die Horvath'sche (1986) Subjektposition nicht identisch sein kann mit der im vorangehenden Kapitel vorgeschlagenen Position [Spec, Top2P], da die Satzadverbialia im Topikbereich nicht den nonspezifischen Indefinita nachfolgen können, wohl aber den Subjekt-NPn.

In Anbetracht des großen Stellenwerts, der Sätzen mit zwei A-Topiks in der Fachliteratur zuteil wird, ist es nicht unwichtig festzuhalten, dass wir diese in unserem Korpus nur in ganz geringer Zahl gefunden haben: 16 Sätze, in denen beide Konstituenten alle Anforderungen des Frey'schen Topiktests bestehen, und 28 Sätze, in denen von zwei nominalen Ausdrücken der zweite das Subjekt ist. Dabei erfüllen solche Subjekte bemerkenswerterweise diese Anforderungen häufig nicht.

\subsubsection{Informationsstatus der A-Topiks im Topikbereich}

Die Frage (23ii) bezog sich auf die Reihenfolge der Topiks mit Informationsstatus gegeben und neu. Von 16 Beispielen, deren zwei präverbale Konstituenten den A-Topik-Test bestehen, habe ich ein einziges Beispiel, nämlich (30), gefunden, in dem sich ein Topik mit dem Informationsstatus neu befindet (a hegyen azóta felépült antennarendszer), dies aber geht im Satz dem anderen Topik mit dem Informationsstatus gegeben voran (a háromszögelési pontot):

(30) A hegyen azóta felépült antennarendszer a háromszögelési art Berg.AUF seitdem aufgebaute Antennensystem ART triangulation pontot megsemmisítette.

Station.AKK vernichtete

'Das auf dem Berg seitdem aufgebaute Antennensystem vernichtete die Triangulation Station.'

Das obige Beispiel widerspricht also Liptáks (2011) bereits zitierter Behauptung, dass Topiks mit dem Informationsstatus neu denen mit Informationsstatus gegeben immer nachfolgen. In den übrigen 15 Beispielen ist die Reihenfolge der beiden A-Topiks in drei Fällen gegeben-gegeben, in einem Fall gegeben-zugänglich, in fünf Fällen zugänglich-gegeben und in vier Fällen zugänglich-zugänglich. 


\subsubsection{Reihenfolge und Interpretation von A-Topiks und temporalen und lokalen Adverbialen}

Die Frage iii) unter (23) bezog sich auf die Reihenfolge von A-Topiks und lokalen und temporalen Adverbialen (als potenziellen R-Topiks). Unter den Sätzen, die zwei Konstituenten vor dem Verb mit Verbpartikel hatten, habe ich 192 gefunden, wo das Adverbial einer A-Topik-Konstituente nachfolgte, und 110, wo deren Reihenfolge die umgekehrte war. Im Zusammenhang damit sind wir auf ein bisher noch nicht beschriebenes, interessantes Phänomen aufmerksam geworden. Nach gewissen Temporaladverben, wie közben 'inzwischen', később 'später', ezután 'dann' usw., besteht das Subjekt den Frey'schen (2004) A-Topik-Test nicht, was daran $\mathrm{zu}$ sehen ist, dass die unten stehenden Zweisatztexte (deren jeweiligen zweiten Sätze aus dem Wikipedia-Korpus stammen) nicht als wohlgeformt betrachtet werden können:

a. Mondok valamit Rimszkij-Korszakovról. erzähle etwas.AKK Rimsky Korsakow.über 'Ich erzähle etwas über Rimsky Korsakow.'

b. \#Közben Rimszkij-Korszakov elveszítette a második inzwischen Rimsky Korsakow verlor ART zweite zürichi csatát.

Zürcher Schlacht.AKK

\# 'Inzwischen hat Rimsky Korsakow die zweite Zürcher Schlacht verloren.'

(32) a. Mondok valamit Miklósról. erzähle etwas. AKK Miklós.über

'Ich erzähle etwas über Miklós.'

b. \# Mindeközben Miklós megpróbál jó apaként viselkedni. inzwischen Miklós versucht gut Vater.wie benehmen.inf 'Inzwischen versucht Miklós sich wie ein guter Vater zu benehmen.'
a. Mondok
valamit
Caesarról.
erzähle
etwas. AKK
Caesar.über
'Ich erzähle etwas über Caesar.'
b. \# Később Caesar megkérdezi Benjamint, mit gondol später Caesar fragt Benjamin.AKK was.AKK meint Johnról. John.über 'Später fragt Caesar Benjamin, was er wohl von John meint.'


Es ist aber interessant, dass sich nicht alle Temporaladverbialen analog zu denen unter (31)-(33) verhalten. Die folgenden Zweisatztexte (wo der jeweilige zweite Satz ebenfalls aus dem Korpus stammt), können wohl als kohärent betrachtet werden:
a. Mondok
valamit
a
nagyhercegröl.
erzähle
etwas. AKK
ART
Großherzog.über

'Ich erzähle etwas über den Großherzog.'

b. Az eljegyzést követően a nagyherceg visszatért ART Verlobung.AKK nachfolgend ART Großherzog zurückkehrte Olaszországba.

Italien.in

'Nach der Verlobung ist der Großherzog nach Italien zurückgekehrt.'

a. Mondok valamit a szovjetekről.

erzähle etwas.AKK ART Sowjets.über

'Ich erzähle etwas über die Sowjets.'

b. Január elejére a szovjetek elérték a háború előtti

Januar Anfang.auf ART Sowjets erreichten ART Krieg vorangehend lengyel határt.

polnisch Grenze.AKK

'Bis Anfang Januar haben die Sowjets die polnische Vorkriegsgrenze erreicht.'

a. Mondok valamit gróf Bethlen miniszterelnökről. erzähle etwas.AkK Graf Bethlen Ministerpräsident.über 'Ich erzähle etwas über Ministerpräsident Graf Bethlen.'

b. A koalíció felbomlásakor gróf Bethlen miniszterelnök ART Koalition Auflösung.bei Graf Bethlen Ministerpräsident aufgreifte a kezdeményezést. felkarolta ART Initiative.AKK 'Während der Auflösung der Koalition hat Ministerpräsident Graf Bethlen die Initiative aufgegriffen.'

Obwohl auf die möglichen Gründe des obigen Kontrasts hier nicht ausführlich eingegangen werden kann, ist es wichtig, einen Unterschied bei den untersuchten Adverbialklassen zu beachten: Während die Sätze unter (34)-(36) von konkreten Zeitpunkten (wenn auch nur auf indirekte Weise) handeln, verhalten sich die anaphorischen Adverbialen in (31)-(33), die das Erzählen irgendeiner Geschichte strukturieren, als würden sie über die Situation insgesamt etwas 
sagen. Wenn Letzteres bedeuten würde, dass wir es mit thetischen Sätzen zu tun haben, dann ist es verständlich, warum die Konstituente in der zweiten Position den Topik-Test nicht besteht.

Es ist interessant, dass wir viel kohärentere Texte erhalten, wenn den anaphorischen Adverbialen, die denen in (31)-(33) ähnlich sind, akkusativische NPn im Topikbereich nachfolgen: ${ }^{30}$

a. Mondok valamit a kanyarról. erzähle etwas. AKK ART Straßenkurve.über

'Ich erzähle etwas über die Straßenkurve.'

b. Később a kanyart átépítették, és róla nevezték el. später ART Straßenkurve.AKK umbauten und nach.ihm benannten PFX 'Die Straßenkurve wurde später umgebaut und wurde nach ihm benannt.'

\begin{tabular}{|c|c|c|}
\hline $\begin{array}{l}\text { Mondok } \\
\text { erzähle }\end{array}$ & $\begin{array}{l}\text { valamit } \\
\text { etwas.AKK }\end{array}$ & $\begin{array}{l}\text { a } \\
\text { ART }\end{array}$ \\
\hline
\end{tabular}

'Ich erzähle etwas über die Leichen.'

b. Ezután a hullákat becsavarták a saját ágynemüjükbe, dann ART Leichen.AKK gewickelten ART eigen Bettücher.in és teherautóval kivitték a közeli erdőbe. und Lastwagen.mit nahmen ART naheliegend Wald.in 'Dann wurden die Leichen in ihre eigenen Betttücher gewickelt und mit Lastwagen in den naheliegenden Wald gefahren.'

\subsubsection{Beobachtungen zu Strategien der Besetzung des Topikbereiches}

Wie oben angemerkt, behauptet É. Kiss (2005), dass es im Fall von transitiven Zustandsveränderungsverben nur dann möglich ist, dass alle Argumente die VP verlassen, wenn entweder eine Konstituente zu Kontrastierungszwecken in die präverbale Fokusposition bewegt oder das Verb kontrastiert oder negiert wird.

Ich habe jedoch unter den Beispielen 44 Sätze gefunden, wo zwei nominale Argumente - meistens Subjekt und (Akkusativ-)Objekt - im Topikbereich vor einem Verb der Zustandsänderung stehen, und festgestellt, dass hinter dem Verb

30 Zur Messung der Kohärenz bräuchten wir natürlich entsprechende empirische Untersuchungen. 
keine Konstituente mehr zu finden war, ohne dass im Kontext ein Kontrast bezüglich der Bedeutung des Verbs festzustellen war.

Unter (39) zeige ich einige Beispiele als Illustration, wo in Klammern der Informationsstatus der Argumente vor dem Verb gekennzeichnet ist:
a. A falut a török elpusztította.
ART Dorf.AKK ART Türke vernichtete
'Die Türken haben das Dorf vernichtet.'
(falut: gegeben, török: zugänglich)
b. Frank a lövéstől elkábul.
Frank ART Schuss.von in.Ohnmacht.fällt
'Vom Schuss fiel Frank in Ohnmacht.'
(Frank: gegeben, a lövéstől: zugänglich)
$\begin{array}{lll}\text { c. Görgey } & \text { Pestet } & \text { kiürítteti. } \\ \text { Görgey } & \text { Pest.AKK } & \text { lässt.evakuieren }\end{array}$
'Görgey lässt Pest evakuieren.'
(Görgey: zugänglich, Pestet: gegeben)

Die obigen Beispiele zeigen, dass die von É. Kiss (2005) formulierten Regelmäßigkeiten über die Positionen der Argumente in Sätzen mit Verben der Zustandsänderung nicht von allgemeiner Gültigkeit sind. Da nach meinen Erfahrungen (wie in Abschnitt 4.3.2 dargestellt) in Sätzen, wo sich sowohl das Subjekt als auch das (Akkusativ-)Objekt im Topikbereich befindet, der Referent der obigen Konstituenten ausschließlich gegeben oder zugänglich sein kann, ergibt sich folgende Erklärung: Der Sprecher stellt diese Konstituenten deshalb an die linke Peripherie des Satzes, damit er den Zusammenhang des Satzes mit früheren Teilen des Textes betont und so die Kohärenz des Textes herstellt. Die Existenz der Beispiele unter (39) beleuchtet also die sonst so triviale Tatsache, dass die Strukturierung eines Satzes innerhalb des Textes auch solche Tatsachen beeinflussen, welche im Falle von isolierten - z.B. auf die Frage Was ist passiert? antwortenden - Sätzen keine Rolle spielen.

\section{Fazit}

Ziel des vorliegenden Beitrags war es, die informationsstrukturellen Eigenschaften der Elemente im Topikbereich zu untersuchen. Bezug nehmend darauf, dass ich in erster Linie den Zusammenhang zwischen der syntaktischen Position und der Funktion des A(boutness)-Topiks untersucht werden sollte, habe ich zuerst 
einen Überblick darüber gegeben, welche Topiktypen die Fachliteratur unterscheidet und welche Methoden es gibt, diese in einem Text zu identifizieren. Dann habe ich mich mit den syntaktischen Eigenschaften und Interpretationsmöglichkeiten der Elemente in der nach syntaktischen Kriterien bestimmten Topikposition des ungarischen Satzes befasst, indem die Feststellungen der relevanten theoretischen Fachliteratur besprochen wurden. Entgegen der bisherigen Annahme eines homogenen Topikfelds im ungarischen Satz wurde auf Grundlage des Stellungsverhaltens von nicht-spezifischen Indefinita und Adverbialen für eine Aufspaltung dieses Felds plädiert.

Schließlich wurden an dem aus Wikipedia-Texten bestehenden Korpus diejenigen Behauptungen aus der Literatur getestet, deren Überprüfung das - bezüglich der Textsorten ziemlich eingeschränkte - Korpus ermöglichte. Während des Testens konnten im Zusammenhang mit den syntaktischen Eigenschaften und Interpretationsmöglichkeiten der Elemente im Topikbereich mehrere neue Beobachtungen gemacht werden, die die Verfeinerung bzw. Modifikation bestehender Theorien notwendig machen. Hierzu gehört das Faktum, dass nicht-Subjekt-NPn als zweite Konstituenten im Topikfeld den Topiktest von Frey (2004) erfüllen. Subjekt-NPn tun dies dahingegen nur, wenn sie auf einen bestimmten Adverbialtyp folgen, aber nie, wenn ihnen eine andere Argument-NP vorangeht. Außerdem konnten klare Abweichungen von den von É. Kiss (2005) gemachten Voraussagen bzgl. der Argumentpositionierung im Topikfeld bei Zustandsveränderungsverben gefunden werden.

\section{Literatur}

Brassai, Sámuel (1860): A magyar mondat. In: Magyar Akadémiai Értesítő. A Nyelv- és Széptudományi Osztály Közlönye 1. 279-399.

Brassai, Sámuel (1963-65): A magyar mondat. In: Magyar Akadémiai Értesítő. A Nyelv- és Széptudományi Osztály Közlönye 3. 3-128, 173-409.

Brentano, Franz (1874): Psychologie vom empirischen Standpunkt. Leipzig: von Duncker und Humblot.

Büring, Daniel (1997): The meaning of topic and focus: The 59th Street Bridge Accent. London/ New York: Routledge.

Büring, Daniel (2003): On D-trees, beans, and B-accents. In: Linguistics and Philosophy 26. 511-545.

Chafe, Wallace (1974): Language and consciousness. In: Language 50. 111-133.

Chafe, Wallace (1976): Givenness, contrastiveness, definiteness, subjects, topics, and point of view. In: Li, Charles (Hg.): Subject and topic. New York: Academic Press. 27-55.

Cook, Philippa/Bildhauer, Felix (2013): Identifying “aboutness topics": Two annotation experiments. In: Dialogue and Discourse 4. 118-141. 
Davidson, Donald (1967): The logical form of action sentences. In: Rescher, Nicholas (Hg.): The logic of decision and action. Pittsburgh: University of Pittsburgh Press. 81-95.

Dipper, Stefanie/Götze, Michael/Skopeteas, Stavros (Hgg.)(2007): Information structure in cross-linguistic corpora: Annotation guidelines for phonology, morphology, syntax, semantics and information structure. Potsdam: Universitätsverlag Potsdam.

Drubig, Hans Bernhard (1992): Zur Frage der grammatischen Repräsentation thetischer und kategorischer Sätze. In: Jacobs, Joachim (Hg.): Informationsstruktur und Grammatik. Opladen: Westdeutscher Verlag. 142-195.

É. Kiss, Katalin (1987): Configurationality in Hungarian. Dordrecht: Reidel Publishing.

É. Kiss, Katalin (1994): Sentence structure and word order. In: Kiefer, Ferenc/É. Kiss, Katalin (Hg.): The syntactic structure of Hungarian. New York: Academic Press. 1-90.

É. Kiss, Katalin (1998a): Identificational focus versus information focus. In: Language 74. 245-273.

É. Kiss, Katalin (1998b): Mondattan. In: É. Kiss, Katalin/Kiefer, Ferenc/Siptár, Péter (Hgg.): Új magyar nyelvtan. Budapest: Osiris. 17-184.

É. Kiss, Katalin (2002): The syntax of Hungarian. Cambridge: Cambrige University Press.

É. Kiss, Katalin (2005): Event types and discourse linking in Hungarian. In: Linguistics 43. 131-154.

É. Kiss, Katalin (2010): An adjunction analysis of quantifiers and adverbials in the Hungarian sentence. In: Lingua 120. 506-526.

Enç, Mürvet (1991): The semantics of specificity. In: Linguistic Inquiry 22. 1-25.

Endriss, Cornelia (2009): Quantificational topics. A scopal treatment of exceptional wide scope phenomena. Dordrecht: Springer.

Farkas, Donka (1994): Specificity and Scope. In: Nash, Léa/Tsoulas, Georges (Hgg.): Actes du Premier Colloque. Langues \& Grammaire. Paris: Université Paris 8. 119-137.

Fodor, Janet D./Sag, Ivan A. (1982): Referential and Quantificational Indefinites. In: Linguistics and Philosophy 5. 355-398.

Frey, Werner (2004): A medial topic position for German. In: Linguistische Berichte $198 \mathrm{i}$. 153-190.

Gécseg, Zsuzsanna/Kiefer, Ferenc (2009): A new look at information structure in Hungarian. In: Natural Language and Linguistic Theory 27. 583-622.

Götze, Michael et al. (2007): Information structure. In: Dipper, Stefanie/Götze, Michael/ Skopeteas, Stavros (Hgg.): Information structure in cross-linguistic corpora: Annotation guidelines for phonology, morphology, syntax, semantics and information structure. Potsdam: Universitätsverlag Potsdam. 147-187.

Gundel, Janet K. (1985): 'Shared knowledge’ and topicality. In: Journal of Pragmatics 9. 83-107. Gyuris, Beáta (2009): The semantics and pragmatics of the contrastive topic in Hungarian. Budapest: The Library of the Hungarian Academy of Sciences and Lexica Publishing.

Gyuris, Beáta (2012): The expression of information structure in Hungarian. In: Krifka, Manfred/ Musan, Renate (Hgg.). 159-186.

Gyuris, Beáta/Mády, Katalin (2014): Contrastive topics between syntax and pragmatics in Hungarian: An experimental analysis. In: Baglini, Rebekah et al. (Hgg.): CLS 46-1: Proceedings of the forty-sixth annual meeting of the Chicago Linguistic Society. The main session. Chicago: CLS. 147-162.

Horvath, Julia (1986): FOCUS in the theory of grammar and the syntax of Hungarian. Dordrecht/ Riverton: Foris Publications.

Jacobs, Joachim (2001): The dimensions of topic-comment. In: Linguistics 39. 641-681. 
Jäger, Gerhard (2001): Topic-comment structure and the contrast between stage level and individual level predicates. In: Journal of Semantics 18. 83-126.

Kadmon, Nirit (2001): Formal pragmatics. Oxford: Blackwell.

Kálmán, László (1985): Word order in neutral sentences. In: Kenesei, István (Hg.): Data and descriptions. (= Approaches to Hungarian 1). Szeged: JATE. 13-23.

Kálmán, László/Nádasdy, Ádám (2004): A hangsúly. In: Kiefer, Ferenc (Hg.): Strukturális Magyar Nyelvtan. Budapest: Akadémiai Kiadó. 393-467.

Kenesei, István (1998): Adjuncts and arguments in VP-focus in Hungarian. In: Acta Linguistica Hungarica 45. 61-88.

Kornfilt, Jaklin (1997): Turkish. London: Routledge.

Krifka, Manfred (2007): Basic notions of information structure. In: Féry, Caroline/Krifka, Manfred (Hg.): Working Papers of the SFB632. Interdisciplinary studies on information structure 6. Potsdam: Universitätsverlag Potsdam. 13-55.

Krifka, Manfred/Musan, Renate (2012): Information structure: Overview and linguistic issues. In: Krifka, Manfred/Musan, Renate (Hgg.). 1-43.

Krifka, Manfred/Musan, Renate (Hgg.)(2012): The expression of information structure. Berlin: de Gruyter.

Kuroda, Sige-Yuki (1972): The categorical and the thetic judgment: Evidence from Japanese. In: Foundations of Language 9.153-185.

Ladusaw, William (1994): Thetic and categorical, stage and individual, weak and strong. In: Harvey, Mandy/Santelmann, Lynn (Hgg.): Proceedings of the 4th Semantics and Linguistic Theory Conference, 6.-8. Mai 1994 (SALT 4). Ithaka/New York: CLC Publications. 220-229.

Lasnik, Howard/Saito, Mamoru (1992): Move- $\alpha$. Cambridge, MA: MIT Press.

Lipták, Anikó (2011): The structure of the topic field in Hungarian. In: Benincà, Paola/Munaro, Nicola (Hg.): Mapping the left periphery. Oxford: Oxford University Press.

Maienborn, Claudia (2001): On the position and interpretation of locative modifiers. In: Natural Language Semantics 9. 191-240.

Marty, Anton (1884): Über subjektlose Sätze und das Verhältnis der Grammatik zu Logik und Psychologie. In: Vierteljahresschrift für wissenschaftliche Philosophie 8. 56-94.

Marty, Anton (1918): Gesammelte Schriften. Halle: Niemeyer.

Molnár, Valéria (1991): Das TOPIK im Deutschen und Ungarischen. Stockholm: Almqvist \& Wiksell International.

Molnár, Valéria (1998): Topic in focus: On the syntax, phonology, semantics and pragmatics of the so-called 'contrastive topic' in Hungarian and German. In: Acta Linguistica Hungarica 45. 89-166.

Molnár, Valéria (2011): ,,Fundamentális” kérdések az információstruktúrában - univerzális és tipológiai megfigyelések tükrében. In: Bartos, Huba (Hg.): Általános Nyelvészeti Tanulmányok XXIII. Új irányok és eredmények a mondattani kutatásban. Budapest: Akadémiai Kiadó. 15-49.

Puskás, Genoveva (2000): Word order in Hungarian. The syntax of A'-positions. Amsterdam/ Philadelphia: Benjamins.

Reinhart, Tanya (1981): Pragmatics and linguistics. An analysis of sentence topics. In: Philosophica 27. 53-94.

Rizzi, Luigi (1997): The fine structure of the left periphery. In: Haegeman, Liliane (Hg.): Elements of grammar. Dordrecht: Kluwer. 281-337. 
Roberts, Cragie (2011): Topics. In: von Heusinger, Klaus/Maienborn, Claudia/Portner, Paul

(Hgg.): Semantics. (= Handbücher zur Sprach- und Kommunikationswissenschaft 33).

Berlin: de Gruyter. 1908-1934.

Sasse, Hans-Jürgen (1987): The thetic/categorical distinction revisited. In: Linguistics 25. 511-580.

Stalnaker, Robert (1978). Assertion. In: Cole, Peter (Hg.): Pragmatics. (= Syntax and Semantics 9). New York: Academic Press. 315-332.

Strawson, Peter (1964): Identifying reference and truth values. In: Theoria 30. 96-118.

Szabolcsi, Anna (1997): Strategies for Scope Taking. In: Szabolcsi, Anna (Hg.): Ways of Scope Taking. Dordrecht: Kluwer. 109-154.

Vallduví, Enric (1990): The informational component. Promotionsschrift, University of Pennsylvania. Philadelphia.

van Hoof, Hanneke (2003): The rise in the rise-fall contour: Does it evoke a contrastive topic or a contrastive focus? In: Linguistics 41. 515-563.

Varga, László (2002): Intonation and stress. Evidence from Hungarian. Basingstoke: Palgrave Macmillan.

Wagner, Michael (2008): A compositional analysis of contrastive topics. In: Abdurrahman, Muhammad/Schardl, Anisa/Walkow, Martin (Hgg.): Proceedings of the North East Linguistic Society 38. Amherst: University of Massachusetts. 415-428.

Ward, Gregory Louis (1985): The semantics and pragmatics of preposing. Promotionsschrift, University of Pennsylvania. Philadelphia. 